\title{
Tendon Regeneration is Possible Even During Subtotal Isolation of the SutureSite
}

\section{ISSN: 2576-8875}

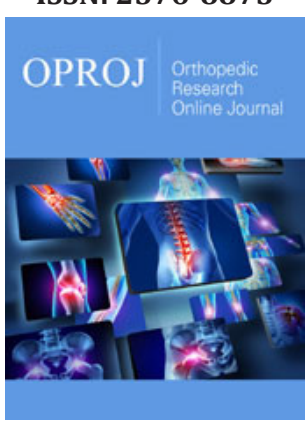

*Corresponding author: Aleksandr V Zenchenko, Department of Orthopedics and Traumatology, Gomel State Medical University, Belarus

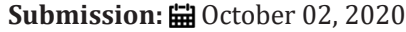

Published: 疅 November 16, 2020

Volume 7 - Issue 4

How to cite this article: Aleksandr $\mathrm{V}$ Zenchenko, Yuliya M Cherniakova. Tendon Regeneration is Possible Even During Subtotal Isolation of the SutureSite. Ortho Res Online J. 7(4). OPROJ. 000670. 2020 DOI: 10.31031/OPROJ.2020.07.000670

Copyright@: Aleksandr V Zenchenko, This article is distributed under the terms of the Creative Commons Attribution 4.0 International License, which permits unrestricted use and redistribution provided that the original author and source are credited.
Aleksandr V Zenchenko ${ }^{1,2 *}$ and Yuliya M Cherniakova ${ }^{2}$

${ }^{1}$ Gomel Regional Clinical Hospital, Belarus

${ }^{2}$ Department of Orthopedics and Traumatology, Gomel State Medical University, Belarus

\section{Opinion}

The process of post-traumatic regeneration of tendons is well known and described in a number of scientific articles [1-3]. There are 3 phases: inflammatory, reparative and remodeling. As a result of activation of extrinsic and intrinsic sources of regeneration, the tendon reaches its original strength 6 weeks after surgery. Afterwards the "maturation" of suture site occurs under the influence of movements for several months.

The problem is the wrist finger flexor tendons delayed reconstruction within fibro osseous canals. Sometime surgeons have a situation when, 3-4 weeks or later after injury in $2^{\text {nd }}$ zone the ends of the flexor digitorum profundus tendon are found and can be sutured with normal tension. However, due to the scarry transformation of the blood clot and the activation of extrinsic sources, the cavity of the fibro-osseous canal is so scar-altered that the only way of reconstruction is two-step tendoplasty. The results of such repair are often unsatisfactory due to the scar process around the repaired tendons and tenogenic contractures formation [4].

We patented and tested in 11 patients a method of mechanical separation of the sliding surfaces of the tendon and its sheath using temporary isolation by dissected biocompatible polymeric tube $[5,6]$. Despite the good functional results morphological examination of the sutured tendons in operated patients is not possible. However, the tendon healing with limited nutrition of the suture site requires its confirmation. Regeneration with subtotal 3/4 diameter isolation and without external immobilization was studied in a 3-week experiment on the Achilles tendons of 10 Wistar rats. All experiments were conducted in accordance with modern ethical standards $[7,8]$. Figure 1 shows the design of experiment.

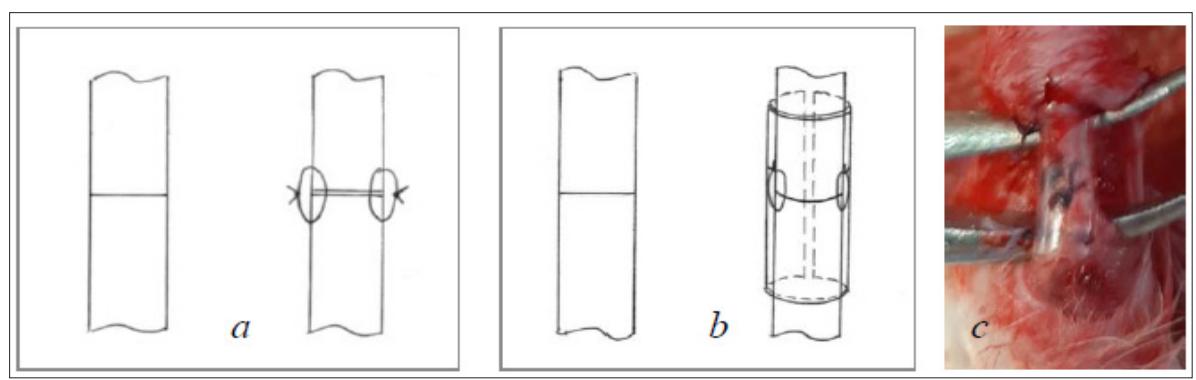

Figure 1: Types of operation on the Achilles tendon: $a$-transverse tenotomy and end-to-end connection (control) on the left paw; $b$-transverse tenotomy, end-to-end connection with dissected tube isolation on the right paw; $c$-view of an isolated tendon.

Tendon preparations were examined at different magnifications using an optical microscope "Levenhuk MED 10T" with digital camera ToupCam 10.0MP (Figure 2). 

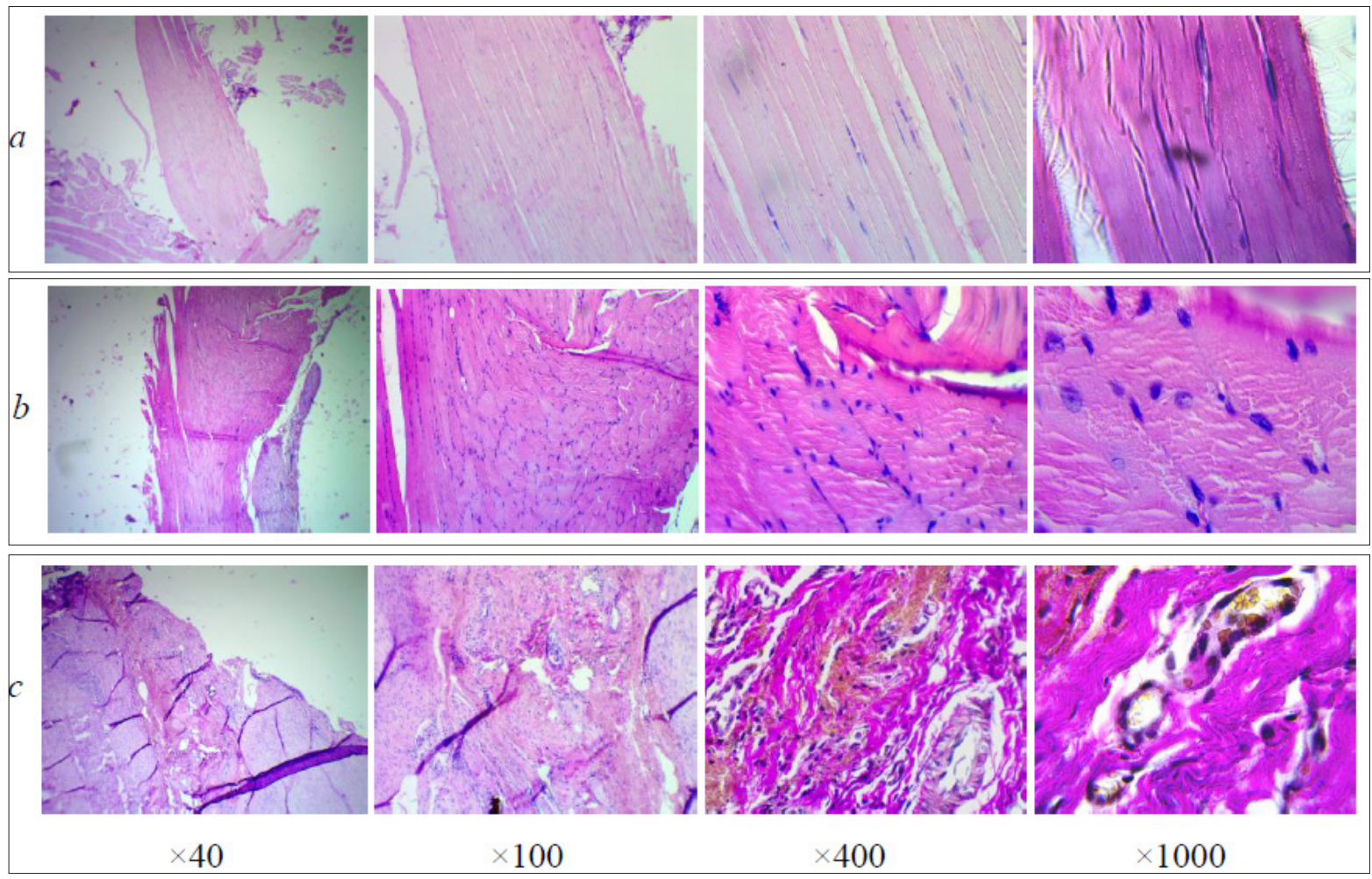

Figure 2: Digital images of tendons: a-intact; b-connected end-to-end; c-after subtotal isolation. Preparations are stained with hematoxylin and eosin, except for the last two in row "c", stained according to Van Gieson.

Comparing rows " $\mathrm{b}$ " и " $\mathrm{c}$ " tendon regenerates are distinguished by their maturity and tissue organization. Better quality tendonlike rearrangement of the suture zone is seen in row "b". The scar includes densely packed collagen fibers and mature tenocytes between fibers and corresponds to the end of the repair phase. At the same time, short and multidirectional fibers in the thickness of the regenerate demonstrate an inadequate rearrangement of the suture site.

Mobilization of the sutured tendon from the first days after the operation did not lead to the formation of sliding surfaces of the tendon and its sheath. In row " $c$ ", the regenerate connects both ends of the tendon over the entire contact area. Its loose structure is represented by richly vascularized tissue, typical of the peak of the repair phase. The metabolic activity of the tendon is manifested by the proliferation of fibroblasts, the presence of histiocytes, macrophages, eosinophils, plasma, lymphoid cells, as well as the appearance of vascular capillaries around the tendon fibers and tendon bundles in contact in the suture site. The surface of the tendon is covered with a thin layer of loose connective tissue (epithenon) that grows on the regenerate. After staining by Van Gieson the first signs of collagen production and its deposition are visible in the form of relatively rare multidirectional short fibers and bundles along the vessels and at the surface of the tendon.

The experiment showed that subtotal tendon isolation affects but does not exclude the healing of the suture site:
1. An early formation of the sliding surfaces of the tendon and the sheath of the tendon sheath took place, which is further important for the free excursion of the tendon and the normalization of movement.

2. Intrinsic tendon healing occurred in conditions of poor nutrition, owing to the metabolic activity of tenocytes and tenoblasts of the tendon itself.

3. Through the dissected area of the tube, connective tissue with blood vessels grew in and neovascularization of the suture area took place.

4. The only negative effect of temporary subtotal tendon isolation was the slowing down of the suture site healing.

For planning motor rehabilitation in clinical practice, it should be remembered about lengthening the reparative period in patients after surgery with the use of isolating tubes.

\section{References}

1. Seiler JG (2001) Flexor tendon repair. J Am Soc Surg Hand 1(3): 177-191.

2. Beredjiklian PK (2003) Biologic aspects of flexor tendon laceration and repair. J Bone Joint Surg Am 85-A(3): 539-550.

3. Gelberman RH, Vandeberg JS, Manske PR, Akeson WH (1985) The early stages of flexor tendon healing: a morphologic study of the first fourteen days. J Hand Surg Am 10: 776-84.

4. Lomaya MP (2005) Causes of fingers tenogenouous flexion contractures formation and their elimination by means of tenolysis (Review). Traum and Orthop of Russia 1(34): 60-67. 
5. Cherniakova YuM, Zenchenko AV, Slepchenko KV (2019) Method of deep finger flexor reconstruction. Patent BY N 22429.

6. Zenchenko AV, Cherniakova YuM (2019) Technology of temporary isolation of the deep digital flexor tendon and management of patients in zone II delayed repair. Genius of Orthopedics 25(3): 290-296.
7. (2002) Institutional Animal Care and Use Committee Guidebook, (2 ${ }^{\text {nd }}$ edn), ARENA.

8. (2011) Guide for the Care and Use of Laboratory Animals, ( $8^{\text {th }}$ edn), National Research Council, National Academy Press, USA.

For possible submissions Click below: 